\title{
Activation of human endogenous retrovirus K (HERV-K) and cellular modifications in human melanoma cell line: transcriptional profiling analysis
}

\author{
Emanuela Balestrieri*1, Claudia Matteucci ${ }^{1}$, Roberta Sorrentino ${ }^{1}$, Reem Al \\ Dossary $^{1}$, Annalucia Serafino ${ }^{2}$, Pasquale Pierimarchi ${ }^{2}$, Gabriella Moroni ${ }^{1}$, \\ Enrico Garaci ${ }^{3}$ and Paola Sinibaldi Vallebona ${ }^{1}$
}

\begin{abstract}
Address: ${ }^{1}$ Department of Experimental Medicine and Biochemical Science, University of Rome "Tor Vergata", Rome, Italy, ${ }^{2}$ Institute of Neurobiology and Molecular Medicine, National Research Council, Rome, Italy and ${ }^{3}$ Istituto Superiore di Sanità, Rome, Italy

* Corresponding author
\end{abstract}

from Frontiers of Retrovirology: Complex retroviruses, retroelements and their hosts Montpellier, France. 21 -23 September 2009

Published: 24 September 2009

Retrovirology 2009, 6(Suppl 2):P4 doi:10.1 I86/I 742-4690-6-S2-P4

This abstract is available from: http://www.retrovirology.com/content/6/S2/P4

(c) 2009 Balestrieri et al; licensee BioMed Central Ltd.

\section{Background}

Similarly to what shown for exogenous tumorigenic retroviruses, endogenous retrovitus (ERVs) have been implicated in the pathogenesis of cancer. ERVs may participate in the process of malignant transformation or promote tumor growth, e.g. through insertional mutagenesis or via counteracting tumor immunosurveillance. Growing evidences show that the activation of endogenous retroviral sequences might be involved in transformation of melanocytes as well as in the increased ability of melanoma cells to escape immune surveillance.

\section{Materials and methods}

The human melanoma cell lines were established in our laboratory as monolayers from two melanoma lesions of the same patient and were maintained in different growth condition to induce cellular modification from adherent to non adherent phenotype. RNA was processed to study ERVs implications and a gene profiling expression analysis was performed to study molecular pathways involved in phenotypic modification.

\section{Results}

We show that human melanoma cells in vitro undergo a transition from adherent to a more malignant, non-adherent phenotype when exposed to stress conditions. These phenotypic and functional modifications are accompanied by the activation of human endogenous retrovirus- $\mathrm{K}$ expression and a massive production of viral-like particles. Down-regulation of HERV-K expression by RNA interference prevents the transition from adherent melanoma to non-adherent phenotype when the human melanoma cell lines are exposed to stress conditions. Melanoma-derived non-adherent cells are characterized by an increased proliferative potential and a decreased expression of both HLA class I molecules and Melan-A/ MART-1 antigen, similarly to highly malignant cells. Super analysis of 440 genes, often modulated during the development and the progression of tumors, shows that many of these genes are modulated during the transition from adherent to non adherent phenotype.

\section{Conclusion}

These results indicate that at least some critical steps of melanoma progression may be HERV-K-dependent. Gene expression analysis highlights an association between viral expression and cellular modification, suggesting a possible role of HERV-K in melanoma phenotypic transformation. 